\title{
Sessile Serrated Adenoma; the Hard-to-Catch Culprit of Interval Cancer
}

\author{
Suk Pyo Shin \\ Department of Internal Medicine, Hallym University College of Medicine, Chuncheon, Korea
}

See "External Validation of the Endoscopic Features of Sessile Serrated Adenomas in Expert and Trainee Colonoscopists" by Hyo-Joon Yang, Jeong In Lee, Soo-Kyung Park, et al., on page 279-286.

Colonoscopic screening and surveillance programs have been implemented in many countries and they have reduced colorectal cancer (CRC)-related deaths. However, this reduction was significantly noted in distal CRC, not in proximal CRC, and some patients may develop unexpected CRC within 3-5 years of colonoscopy. Interval CRCs, which have a prevalence ranging from $1.8 \%$ to $9.0 \%$, are CRCs that are diagnosed after a screening or surveillance examination and before the date of the next recommended exam. It is known that interval CRCs are more common in the proximal colon than in the distal colon. ${ }^{1}$ Research regarding the molecular profiles of interval CRCs and serrated lesions have identified a molecular similarity between the interval CRCs and the pathway for serrated adenoma carcinogenesis. Due to the sessile or flat features associated with these lesions, preference for right colon and molecular features of sessile serrated adenomas/polyps (SSA/Ps), SSA/Ps have been getting attention for their clinical importance in interval CRCs. ${ }^{2,3}$

Despite advancement in imaging systems and some known endoscopic features characteristic of SSA/Ps, the detection and accurate identification of SSA/Ps during colonoscopy is

Received: April 1, 2017 Accepted: April 26, 2017

Correspondence: Suk Pyo Shin

Department of Internal Medicine, Chuncheon Sacred Heart Hospital, Hallym University College of Medicine, 77 sakju-ro, Chuncheon 24253, Korea

Tel: +82-33-240-5823, Fax: +82-33-255-4291, E-mail: ppyo1029@gmail.com

cc This is an Open Access article distributed under the terms of the Creative Commons Attribution Non-Commercial License (http://creativecommons.org/ licenses/by-nc/3.0) which permits unrestricted non-commercial use, distribution, and reproduction in any medium, provided the original work is properly cited. challenging to the colonoscopist.

In this issue of Clinical Endoscopy, Yang et al. ${ }^{4}$ validated previously reported endoscopic features of SSA/Ps and identified features that can be reliably used for SSA/P prediction by experts and trainees. The endoscopic features of SSA/Ps in this study are: indistinct borders, irregular shape, rim of debris, cloud-like surface, mucous cap, nodular surface, absence of surface vessels, and dark spots. Among these eight features, four included independent predictive features for SSA/P histology (indistinctive borders, mucous cap, cloudlike surface and dark spots). Additionally, three of these four features showed moderate interobserver agreement among experts and trainees (the exception being dark sports). These three characteristics rendered $79.0 \%$ sensitivity and $81.4 \%$ specificity for SSA prediction using high resolution white light endoscopy.

With regards to the method of this study, I noticed an interesting point. Yang et al. ${ }^{4}$ held a training session and consensus meeting before the validation of the endoscopic features. This process might be the major reason why the interobserver agreement among trainees was not inferior to that of experts. Therefore, setting up courses that include a training session and consensus meeting might be helpful for trainees, not only for SSA/Ps but also for other lesions. Although the suitable degree of interobserver agreement in trainees in this study could not be applied to other medical centers which have not set-up the courses, the three simple endoscopic features could easily be used and prove to be helpful for colonoscopy training elsewhere. 
Characteristic histologic features of SSA/P are a saw-tooth appearance involving the entire length of the crypt, dilated and/or branched crypts, horizontal extensions of the crypt bases, and herniation of crypts through the muscularis mucosa.

Despite these well-established and generally accepted histologic features, there is substantial interobserver variation among pathologists in the diagnosis of SSA. ${ }^{5}$ Furthermore, there is some disagreement regarding the definition of SSA/P. In the World Health Organization (WHO) classification, diagnosis of SSA is made when there are two or more contiguous SSA-type crypts, ${ }^{6}$ whereas, the Japanese Society for Cancer of the Colon and Rectum (JSCCR) defined an SSA as at having least two SSA-type crypts in $\geq 10 \%$ of the area of the lesion. ${ }^{7}$ In addition, review and recommendations from an expert panel specify that the presence of at least one unequivocal SSA-type crypt is sufficient for a diagnosis of SSA/P. ${ }^{8}$

In this study, the histologic diagnosis of SSA/P was made using the $\mathrm{WHO}$ criteria. If the definition of histologic diagnosis of SSA/P was changed to other criteria, the results of this study might be also influenced.

As seen in this and other studies, ${ }^{9}$ it is unsatisfactory to identify SSA/Ps using characteristic endoscopic features. However, there are several reasons why we must make a steadfast endeavor to differentiate SSA/Ps from non SSA/Ps. First, the suspicion or identification of SSA/Ps during colonoscopy enables the colonoscopist to give more information to the pathologist. In cases of superficial biopsies or electrocautery artifacts, this information would be helpful. Second, when multiple SSA/Ps are suspected during colonoscopy, a more accurate diagnosis of serrated polyposis syndrome, which has been under-recognized and consequently under-managed, could be made.

Given the substantial interobserver variation among pathologists in the differentiation of SSA/Ps from hyperplastic polyps, and the risk for carcinogenesis of SSA/Ps, we should consider proximal serrated lesions $>1 \mathrm{~cm}$ in size to be SSA/Ps. Therefore, an effort to find serrated lesions should be emphasized in current clinical practice rather than to simply identify the SSA/Ps.

In the future, the importance of identification of the SSA/Ps will be markedly emerging.

Conflicts of Interest

The author has no financial conflicts of interest.

\section{REFERENCES}

1. Singh S, Singh PP, Murad MH, Singh H, Samadder NJ. Prevalence, risk factors, and outcomes of interval colorectal cancers: a systematic review and meta-analysis. Am J Gastroenterol 2014;109:1375-1389.

2. Sawhney MS, Farrar WD, Gudiseva S, et al. Microsatellite instability in interval colon cancers. Gastroenterology 2006;131:1700-1705.

3. Arain MA, Sawhney M, Sheikh S, et al. CIMP status of interval colon cancers: another piece to the puzzle. Am J Gastroenterol 2010;105:11891195.

4. Yang HJ, Lee JI, Park SK, et al. External validation of the endoscopic features of sessile serrated adenomas in expert and trainee colonoscopists. Clin Endosc 2017;50:279-286.

5. Gill P, Wang LM, Bailey A, East JE, Leedham S, Chetty R. Reporting trends of right-sided hyperplastic and sessile serrated polyps in a large teaching hospital over a 4-year period (2009-2012). J Clin Pathol 2013;66:655-658.

6. Snover DC, Ahnen DJ, Burt RW, Odze RD. Serrated polyps of the colon and rectum and serrated polyposis. In: Bosman FT, Carneiro F, Hruban $\mathrm{RH}$, Theise ND, eds. WHO classification of tumours of the digestive system. 4th ed. Lyon: International Agency for Research on Cancer; 2010. p. 160-165.

7. Yao T, Sugai T, Iwashita A, et al. Histopathological characteristics and diagnostic criteria of SSA/P, project research 'potential of cancerization of colorectal serrated lesions' of Japanese society for cancer of the colon and rectum. Stomach and Intestine (Tokyo) 2011;46:442-448.

8. Rex DK, Ahnen DJ, Baron JA, et al. Serrated lesions of the colorectum: review and recommendations from an expert panel. Am J Gastroenterol 2012;107:1315-1329; quiz 1314, 1330.

9. Hazewinkel Y, López-Cerón M, East JE, et al. Endoscopic features of sessile serrated adenomas: validation by international experts using high-resolution white-light endoscopy and narrow-band imaging. Gastrointest Endosc 2013;77:916-924. 